\title{
Research on Harmonic Suppression of Direct Drive Permanent Magnet Wind Converter
}

\author{
Shan Li, Rulong Yang, Shuxi Liu* and Lei Tong \\ School of Electrical \&Electronic Information Engineering, Chongqing University of Technology, No.69, Hong-guang Road, \\ District of Ba-nan, Chongqing, China \\ ${ }^{*}$ Corresponding author
}

\begin{abstract}
With the increasing of wind power installed capacity, the demand for power generation quality of wind power system is getting higher and higher. The magnitude of harmonic generation in wind power system plays a decisive role in the output power quality. Effective suppression of harmonics is a key indicator of the quality and performance of wind power converter equipment. Based on the harmonic of direct drive permanent magnet wind power, the high frequency and low frequency character of grid side converter is analyzed. In order to restrain the effect of harmonics, the system adopts active damping and PIR control technology on the harmonic suppression control strategy, so as to improve the quality of the power grid. Through the related experiments on the industrial site, the results show that the control strategy can effectively suppress the harmonic current, and has good feasibility.
\end{abstract}

Keywords—harmonic suppression; active damping; PIR control

\section{INTRODUCTION}

The direct drive permanent magnet wind turbine system has no gear box structure, no brush and slip ring, which makes the unit noise reduction. The space cost reduction and maintenance costs are also greatly reduced. Especially at low wind speeds, the efficiency of the unit's power generation is very obvious [14].

However, the direct-driven wind turbine will generate harmonic interference in the power grid during operation, which will not only cause serious damage to the power equipment of the power system, reduce the service life of the equipment, and may also cause serious production operation accidents to the power plant and users. In order to ensure the quality of power supply and improve the safety and economy of wind power system operation, the harmonic suppression of wind power system has become an important research topic [57].

In this paper, the high-frequency characteristics and lowfrequency characteristics of the direct-drive permanent magnet wind power converter are analyzed. In the harmonic suppression control strategy, by using active damping and PIR control technology, the harmonic current accessing to the power grid is decreased. Then the power quality is improved [8-9]. The experimental results show that this control scheme plays a good role in harmonic suppression.

\section{Direct-Drive Permanent Magnet Wind PoWer CONVERTER}

\section{A. High Frequency Harmonic Characteristics of Line Side Converter}

The grid-side converter is a typical three-phase voltage-fed PWM rectifier. The harmonic expression of the output phase voltage is closely related to the modulation strategy. The SVPWM modulation strategy is adopted in the model to analyze the harmonic model of the output phase voltage.

The diagram of space voltage vector is shown in Fig. I, a total of eight voltage vectors in the figure $\left(U_{1 \sim} U_{6}\right.$ length of $2 U_{d c} / 3, U_{d c}$ is DC voltage of PWM rectifier, $U_{7}$ and $U_{8}$ are zero vector, denoted by $U_{0}$ )corresponds to 6 switch status, respectively. The topology of PWM converter is shown in Fig. II.

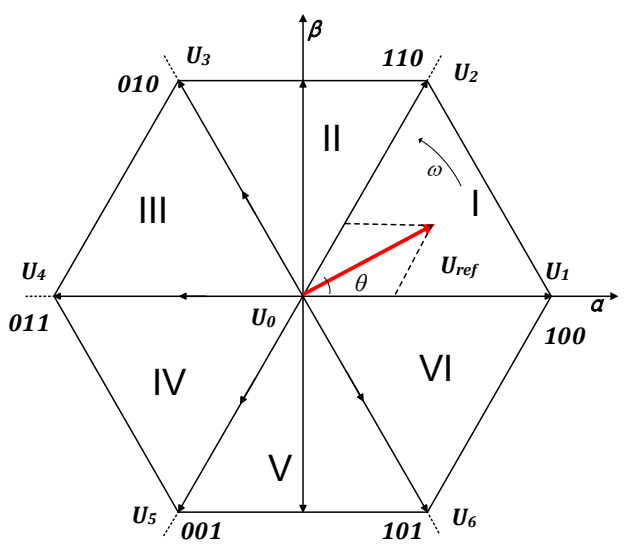

FIGURE I. VOLTAGE SPACE VECTOR

In Fig. I, $U_{r e f}$ is the reference voltage, $\theta_{\text {is }}$ its rotation angle, $U_{r e f}$ can be represented by voltage vector $U_{1} U_{2}$ and $U_{0}$, then the expression of $U_{r e f}$ is as follows:

$$
U_{r e f} \cdot T_{s}=U_{1} T_{1}+U_{2} T_{2}+U_{0} T_{0}
$$


Among them, $T_{s}$ for the switching cycle, $T_{1}$ is the acting time of $U_{1}, T_{2}$ is the acting time of $U_{2}$. The decomposition $U_{\text {ref }}$ can be obtained:

$$
\left\{\begin{array}{c}
U_{r e f} T_{s} \cos \theta=\frac{2}{3} U_{d c} T_{1}+\frac{2}{3} U_{d c} T_{2} \cos 60^{\circ} \\
U_{r e f} T_{s} \sin \theta=\frac{2}{3} U_{d c} T_{2} \sin 60^{\circ}
\end{array}\right.
$$

By the modulation ratio $m_{u}=\sqrt{3} U_{r e f} / U_{d c}$ available:

$$
\left\{\begin{array}{c}
T_{1}=m_{u} T_{s} \sin \left(60^{\circ}-\theta\right) \\
T_{2}=m_{u} T_{s} \sin \theta
\end{array}\right.
$$

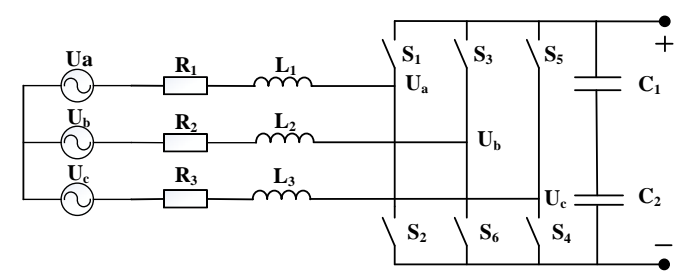

FIGURE II. THE NETWORK TOPOLOGY OF CONVERTER

$U_{a}$ is the voltage which phase a relative to the capacitor midpoint. In a switching cycle $\mathrm{T}$, the positive level duty cycle $\mathrm{D}$ is:

$$
D=\frac{1+m_{u} \cos \left(\theta-30^{\circ}\right)}{2}
$$

Let $f_{s}$ be the switching frequency and $f$ be the output frequency. If the carrier ratio $m_{f}=f_{s} / f$, then the rotation angle step is $d \theta=360^{\circ} / m_{f}$ and the fundamental angular frequency $\omega_{1}=2 \pi f$. Since $U_{a}$ is in one cycle, D $=0.5$, if $a_{0}=0, b_{0}=0$, the Fourier decomposition of $U_{a}$ can be obtained:

$$
U_{a}(t)=a_{0}+\sum_{n=1}^{\infty}\left[a_{n} \cos \left(n \omega_{1} t\right)+b_{n} \sin \left(n \omega_{1} t\right)\right]
$$

From the above formula can be derived:

$$
a_{n}=\frac{4}{T} \int_{0}^{T / 2} U_{a 0}(t) \cos n \omega_{1} t d t
$$

The $T / 2$ cycle is divided into $1+m_{f} / 2$ intervals, the length of the interval $1+m_{f} / 2$ is $T_{s} / 2$, and the interval length is $T_{s}$. If the duty cycle of the interval $\mathrm{k}$ is $d_{u(k)}$, the rotation angle $\theta_{k}$ corresponding to the interval $\mathrm{k}$ is:

$$
\theta_{k}=\frac{(k-1) \cdot 360^{\circ}}{m_{f}}\left(k=1,2, \ldots 1+m_{f} / 2\right)
$$

In $\mathrm{T} / 2$, pulses are respectively given at time points of $t_{0}, t_{1}, \ldots, t_{m_{f}}, t_{m_{f}+1}$, and the corresponding pulse jump points are integrated and developed in a half cycle $T$ / 2 :

$$
\begin{gathered}
a_{n}=\frac{4}{T} \int_{0}^{T / 2} U_{a 0}(t) \cos \left(n \omega_{1} t\right) d t \\
=\frac{2 U_{d c}}{n \pi}\left[\sum_{k=1}^{m_{f} / 2} \sin \left(n \omega_{1} t_{2 k-1}\right)\right. \\
\left.-\sum_{k=2}^{1+m_{f} / 2} \sin \left(n \omega_{1} t_{2 k-2}\right)\right]
\end{gathered}
$$

According to (8), we can get the amplitude of each order harmonic of $U_{a}$, and the output voltage $U_{a n}$ of grid-side converter is

$$
\begin{gathered}
u_{a n}=U_{a}-U_{n o} \\
U_{n o}=\left(U_{a o}+U_{b o}+U_{c o}\right) / 3
\end{gathered}
$$

Among them, $U_{n o}$ is the voltage of the load to the DC capacitor midpoint, because of symmetry for phase abc to capacitive center point, then $U_{n o}$ only contains $3 n$ harmonics, and the amplitude is equal to $3 n$ harmonic amplitude of $U_{a o}$.

When the grid-side converter is in stable operation, if the modulation ratio is constant, the output harmonic amplitude is also constant. Its network-side LCL filter with high-band harmonic model is shown in Fig.3, in which, $L_{3}$ and $L_{4}$ for the filter inductor, $R_{s}$ for damping resistor, ${ }^{C_{f}}$ for the filter capacitor. 


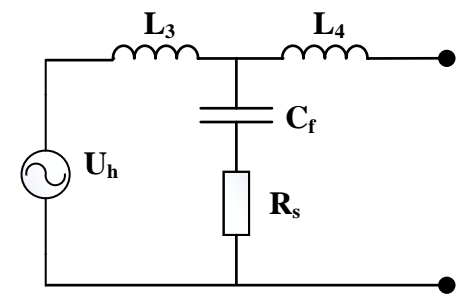

FIGURE III. HIGH-BAND HARMONIC EQUIVALENT MODEL

\section{B. Line-Side Converter Low-Frequency Harmonic Characteristics}

The harmonic characteristics of the grid-side converter in the low frequency band are largely affected by the controller, showing more complex of the characteristics. It can be seen from Fig. IV that the output phase voltages $U_{a}, U_{b}$ and $U_{c}$ of the grid-side converter are determined by the feedback inductor current and the command current by PI control. Since the grid voltage affects the output of the grid-side converter phase voltage in the feed-forward branch, this causes the phase voltage of the grid-side converter to exhibit a controlled voltage source characteristic for harmonics.

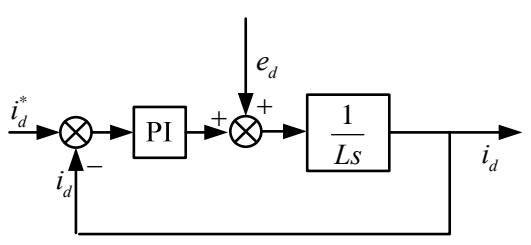

FIGURE IV. CURRENT LOOP CONTROL

For low frequencies, because power electronics elements are not ideal components, different nature and characteristics of harmonics are produced during switching. Converter consists of a large number of power electronic components, the resulting large number of harmonics cannot be ignored. Otherwise it will directly affect the quality of the output power. Therefore, the harmonic produced by the power electronics of the converter cannot be neglected. This part of the harmonic generation is not ideal, it can be regarded as an independent voltage source. Since the harmonic impedance directly affects the magnitude of the harmonic voltage, the controller has the greatest impact on the impedance characteristics.

The analysis of low frequency harmonic characteristics of grid side converter is shown in Fig. V, where $C_{I}(s)$ and $C_{v}(s)$ are feed-forward transfer functions of current and voltage loop respectively, $U_{T}$ is the harmonic generated by non ideal components, and $U_{c}$ is the controlled voltage source controlled by voltage loop and current loop.

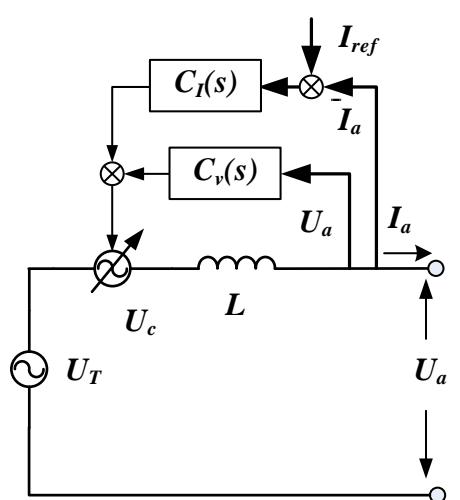

FIGURE V. ANALYSIS OF LOW FREQUENCY HARMONIC CHARACTERISTICS

The converter inductance $\mathrm{L}$ is equivalent to a whole, and when the outside of the inductor is open, $I_{a}=0$, the open circuit voltage $U_{o}$ can be obtained by the thevenin equivalent principle.

$$
\begin{gathered}
U_{o}(h)=U_{T}(h)+U_{c}(h) \\
U_{c}(h)=-I_{a}(h) \cdot C_{I}(h)+C_{v}(h) \cdot U_{a}(h) \\
\text { From } U_{a}(h)=U_{o}, \text { it can be obtained: } \\
U_{o}(h)=\frac{U_{T}(h)}{1-C_{v}(h)}
\end{gathered}
$$

If $U_{T}=0$, the harmonic impedance is:

$$
Z_{e q}(h)=\frac{C_{I}(h)+Z_{L}(h)}{1-C_{v}(h)}
$$

\section{HARMONIC SUPPRESSION STRATEGY}

\section{A. Active Damping}

The direct drive permanent magnet grid filter can be divided into two types of single inductor and LCL. Compared to the single inductor filter, high frequency harmonic filtering ability of LCL filter has better filtering effect, in the same circumstances, the total inductance of LCL filter which needs much less than the single inductor filter, not only reduce the volume and weight of the converter main circuit, but also greatly reduces the cost.

According to the basic principle of the circuit, we know that the resonant frequency exists in the LCL circuit, the frequency 
response has resonance peak at the resonance frequency, and the phase will occur -180 degree jump. The single-phase LCL circuit shown in Fig.6 is composed of $L_{5}, C_{5}$ and $L_{6}$. The $L_{5}$, corresponds to the reactance of the direct drive permanent magnet converter, the $C_{5}$ corresponds to the capacitor filter circuit, and the $L_{6}$ corresponds to the network side inductance. In the ideal case, the transfer function of the inverter output $U_{\text {in }}$ to the network side current ${ }^{L_{6}}$ can be derived as follows:

$$
G(s)=\frac{1}{\left[L_{5} L_{6} C_{5} s+\left(L_{5}+L_{6}\right) s\right]}
$$

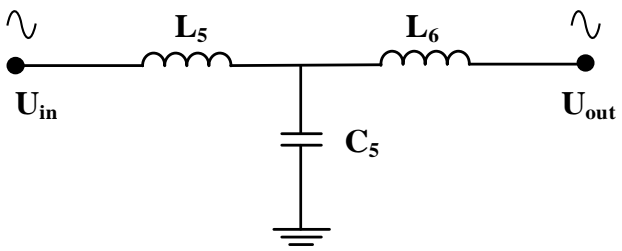

FIGURE VI. SINGLE-PHASE LCL FILTER

By the LCL transfer function, the resonant frequency can be obtained.

$$
\omega_{\text {res }}=\sqrt{\frac{L_{5}+L_{6}}{L_{5} L_{6} C_{5}}}
$$

Set $L_{5}=75 \mu H, L_{6}=50 \mu H \quad C_{5}=668.4 \mu F$, and the frequency characteristics of the system are shown in Fig.VII. When the operation frequency is $1.12 \mathrm{kHz}$, it is known from Fig.7 that the angle margin of the system satisfies the stability criterion, but there is a certain resonant peak.

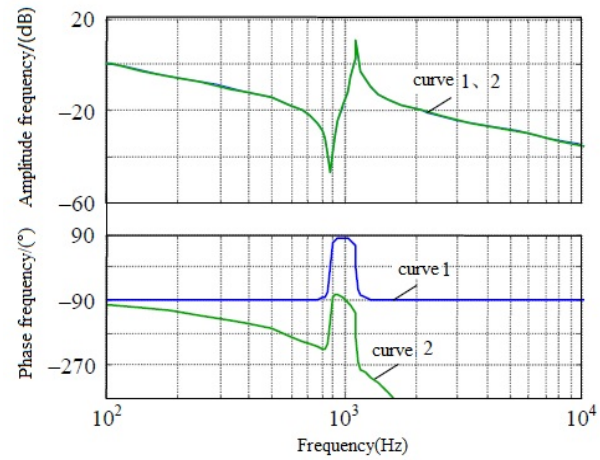

FIGURE VII. FREQUENCY CHARACTERISTIC CURVE

When the operation frequency is $3.2 \mathrm{kHz}$, the phase margin of the system is not satisfied with the stability criterion. The lag of the real control system, and the amplitude and frequency gain of the system is very small, which makes the system divergence. The capacitance series resistance can restrain the effect of resonance to a certain extent, but this will increase the loss of the system.

Therefore, the active damping method using virtual resistance is a very effective method. The essence is to use the control algorithm instead of the actual resistance, so as to achieve the damping effect to effectively suppress the resonant peak. There are two main methods, which are virtual resistance series capacitor and parallel capacitor. Because the series capacitance is too much, it can produce noise. Therefore, the use of virtual resistance parallel capacitance is the best. It is assumed that the value of the virtual resistance is $R_{d}$, and the transfer function of the system after the parallel virtual resistance is as follows:

$$
\begin{aligned}
G^{\prime}(s)= & L_{2} C_{f}\left(R_{d}+R\right) s^{2}+\left(L_{2}+R_{d} R C_{f}\right) s \\
& +R_{d} /\left\{L_{1} L_{2} C_{f}\left(R_{d}+R\right) s^{2}\right. \\
& +\left[L_{1} L_{2}+\left(L_{1}+L_{2}\right) R R_{d} C_{f}\right] \\
& \left.+\left(L_{1}+L_{2}\right) R_{d} s\right\}
\end{aligned}
$$

When the $R_{d}$ is 0.75 times the capacitance resistance the frequency characteristics is shown curve 2 in Fig. VIII; when 0.25 times the capacitance resistance, the frequency characteristics is shown curve 3 in Fig.8. Among them, the curve 1 is the frequency characteristic obtained without damping. We can see from Fig. VIII, the $R_{d}$ can inhibit resonant peaks, but in practical applications, $R_{d}$ is not the bigger the better, the general selection for 0.25-0.75 times of the capacitor capacitance.

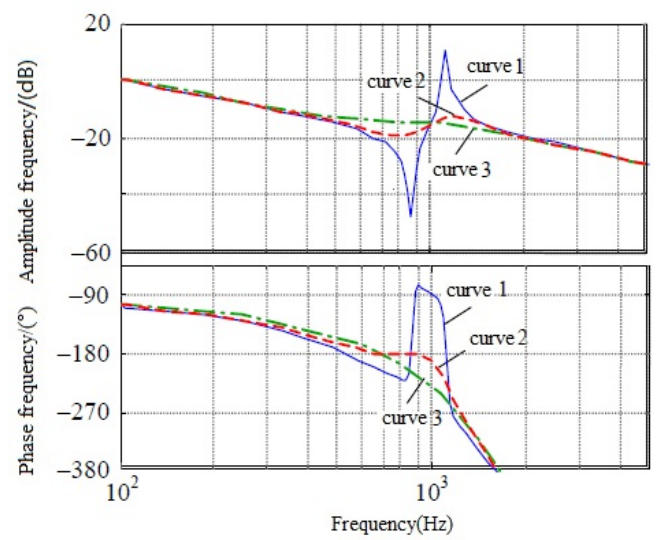

FIGURE VIII. FREQUENCY CHARACTERISTICS OF DIFFERENT VIRTUAL RESISTANCE VALUES.

\section{B. Pir Control}

In the wind power generation unit, the 5 or 7 harmonic currents inevitably occur in the power electronic converters themselves, which make the 5 and 7 times harmonic voltage of the net-dot voltage larger, and ultimately lead to the network current can not meet the power quality requirements. Neither 
the L filter nor the LCL filter can suppress the low order harmonics. For the generator, its low harmonic impedance is small, and the suppression of low order harmonic is also very limited. A harmonic suppression strategy based on resonant controller is adopted to deal with the 5 and 7 harmonics. The scheme does not need to extract the specific sub-harmonic content. It only needs to add a resonant controller and occupy less resources, which is also convenient and cost saving in practical applications.

The bode diagram of PIR controller is shown in Fig. IX. Because grid voltage can not be changed, grid side converter adopts grid voltage oriented vector control strategy. By controlling the voltage of converter's AC side, active and reactive components of input current can be controlled. The 5 harmonic components and 7 harmonic components of the power grid harmonics are mainly represented by the 6 harmonic components in the forward synchronous rotating coordinate system of the basic frequency of the power grid. Therefore, 6 harmonic controllers can be added to the device to suppress the 5 and 7 harmonic components. The 6 times resonant control controller is added, which improves the gain of PI control at fixed frequency point $(300 \mathrm{~Hz})$, does not change the gain and phase of other frequency points, and has little influence on the stability of the system.

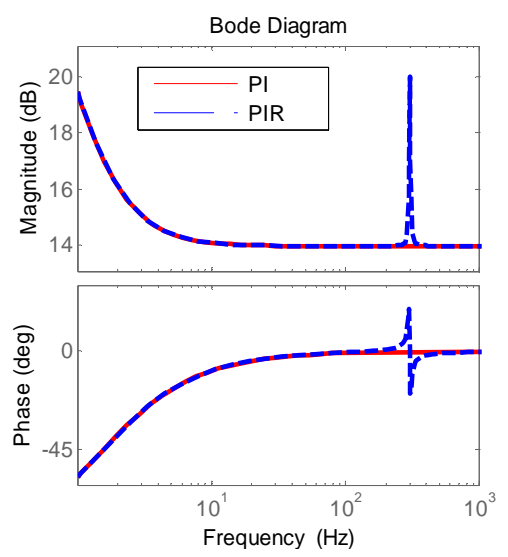

FIGURE IX. PIR CONTROL AND PI CONTROL DIAGRAM

The control of the 6 stage resonant controller is mainly composed of three parts as shown in Fig.10. One is the output of the conventional PI regulator, the two is the output of the 6 resonant controller, and the three is the DQ axis coupling term and the feed forward quantity.

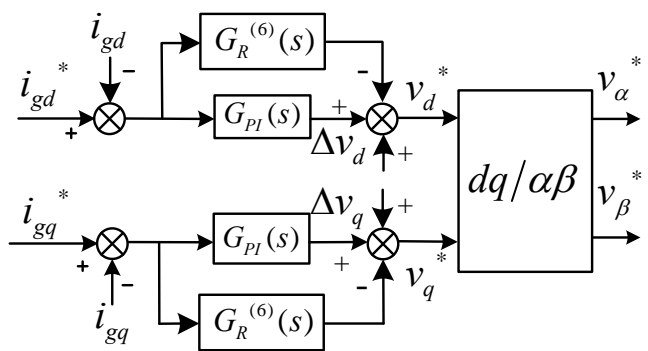

FIGURE X. BLOCK DIAGRAM OF INNER LOOP CONTROLLER
The transfer function of the regulator and the 6 resonant controller can be expressed as:

$$
G_{P I}(s)=K_{p}+\frac{K_{i}}{s}
$$

$$
G_{R}{ }^{(6)}(s)=\frac{2 K_{r}{ }^{(6)} \omega_{c} s}{s^{2}+2 \omega_{c} s+\left(6 \omega_{s}\right)^{2}}
$$

Among them, ${ }^{K_{p}}$ and $K_{i}$ are PI regulator proportions and integration coefficients. $K_{r}^{(6)}$ is the gain of 6 resonant controller, $\omega_{c}$ is the width of controller bandwidth ( $\left.\mathrm{rad} / \mathrm{s}\right)$, and ${ }_{6} \omega_{s}$ as the resonant frequency ( $\left.\mathrm{rad} / \mathrm{s}\right)$. For the actual controller, it is necessary to use the digital control technology to realize the resonant controller. With the help of Matlab software, the resonant controller is dispersed. The sampling frequency is $f_{s}=2900 \mathrm{~Hz}$, the gain coefficient of the 6 time resonant controller is $K_{r}^{(6)}=1$, and the bandwidth $\omega_{c}=5 \pi \mathrm{rad} / \mathrm{s}$. The bode diagrams of three kinds of discretization methods are given in Fig.11, it can be seen in the vicinity of the center frequency of $300 \mathrm{~Hz}$, the impulse response method is closest to the transfer function characteristics and continuous domain, and bilinear transformation method of center frequency is of offset. Zero-order hold method will produce phase lag effect. This method is used to discretize the controller because of the best result of the impulsive response method.

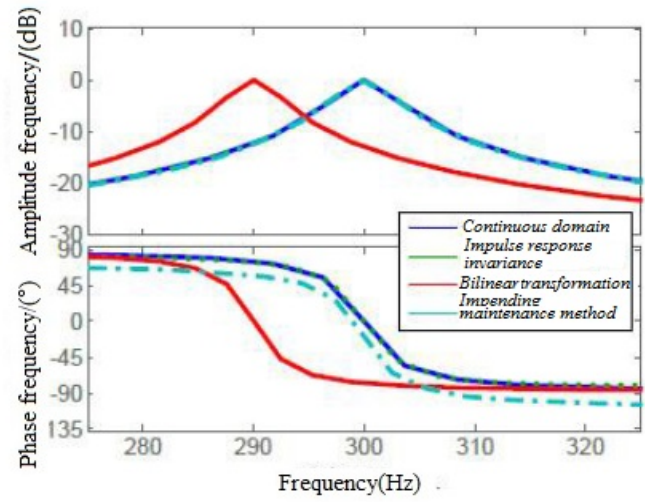

FIGURE XI. CONTRAST DIAGRAM OF FREQUENCY CHARACTERISTICS OF DIFFERENT DISCRETIZATION METHODS 


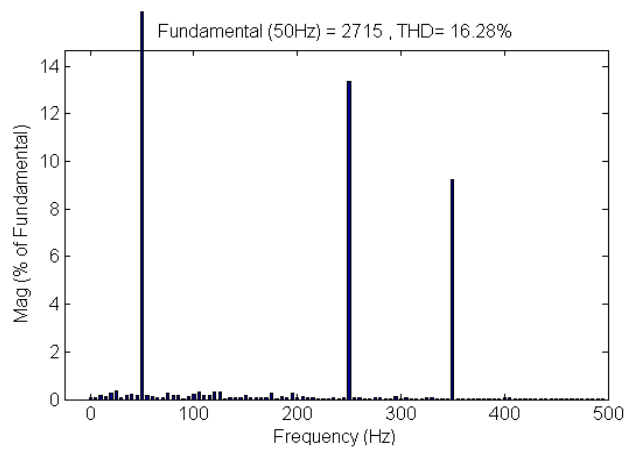

FIGURE XII. CONVENTIONAL PI CONTROL

The simulation results show that the 6 harmonic controller can effectively reduce the 5 and 7 harmonic currents and do not amplify the other harmonics. It is shown in Fig. XII and Fig. XIII.

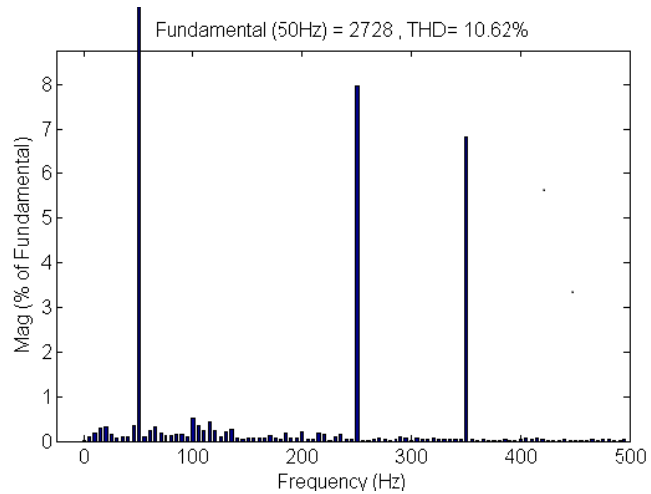

FIGURE XIII. 6 TIMES RESONANT CONTROLLER ON THE SIDE OF THE NET

\section{EXPERIMENTAL VERIFICATION}

At the site of a $2 \mathrm{MW}$ wind power project, the related experiments were carried out on the converter. From the comparison of Fig. XIV and Fig. XV, the $1020 \mathrm{~Hz}$ resonant peak is suppressed after the active damping is put into.

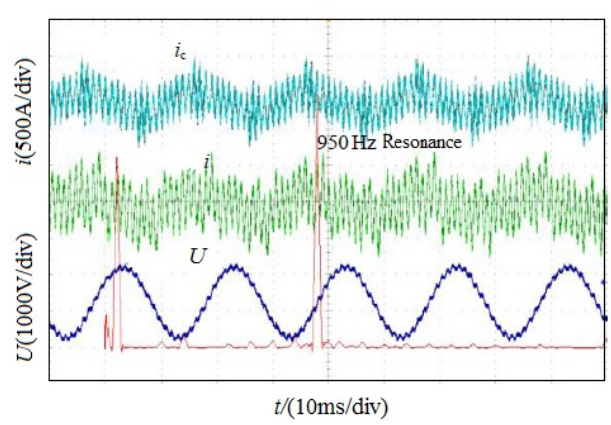

FIGURE XIV. 1020HZ RESONANT WAVEFORM

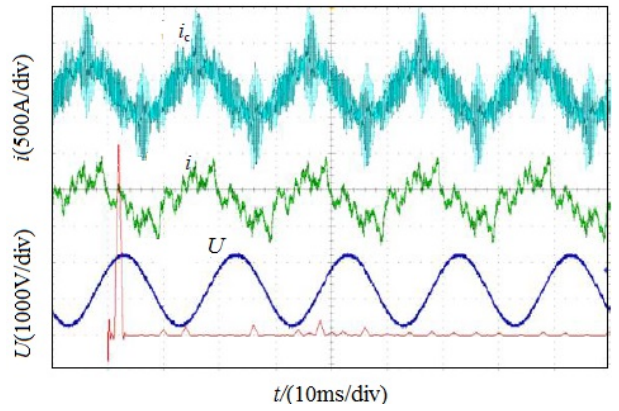

FIGURE XV. 1020 RESONANT WAVEFORMS AFTER ACTIVE DAMPING INPUT

After the 6 resonant controller is put into the system, the 5 and 7 harmonics and the net current THD are greatly reduced. It is shown in Table I.

TABLE I. THE COMPARED PARAMETERS WITH DIFFERENT CONTROL

\begin{tabular}{ccccc}
\hline & $\begin{array}{c}\text { Amplitude } \\
\text { of current } \\
\text { fundament } \\
\text { al wave } \\
\text { (A) }\end{array}$ & $\begin{array}{c}\text { Percenta } \\
\text { ge of 5 } \\
\text { times } \\
\text { harmoni } \\
\text { cs }\end{array}$ & $\begin{array}{c}\text { Percent } \\
\text { age of 7 } \\
\text { times } \\
\text { harmon } \\
\text { ics }\end{array}$ & $\begin{array}{c}\text { Net } \\
\text { current } \\
\text { THD }\end{array}$ \\
\hline $\begin{array}{c}\text { Conventional } \\
\text { PI control }\end{array}$ & 2715 & $13.7 \%$ & $9.4 \%$ & $16.3 \%$ \\
\hline $\begin{array}{c}\text { 6times } \\
\text { resonant } \\
\text { controller on } \\
\text { the side of the } \\
\text { net }\end{array}$ & 2728 & $8 \%$ & $6.8 \%$ & $10.6 \%$ \\
\hline
\end{tabular}

\section{CONCLUSION}

This paper analyzes the harmonic characteristics of the direct drive permanent magnet converter at high and low frequencies, and then uses active damping and PIR control technology to suppress harmonic. The industrial field test proves that the active damping method using virtual resistor can suppress the peak of the system resonance very well, and the harmonic of the output current of the system can be greatly reduced by the PIR control technology, which proves the effectiveness and feasibility of the control scheme.

\section{REFERENCES}

[1] Liu Cheng. Research on embedded AC permanent magnet synchronous motor servo control system based on DSP [D]. Northeast Normal University, 2009.

[2] Qu Xinghong. Direct drive permanent magnet synchronous wind power control system research and design of [D]. Chongqing University, 2008.

[3] Mi Fuli Direct drive wind power system harmonic resonance and [D]. of Harbin Institute of Technology, 2013.

[4] Chen Rong. Design of current ring of permanent magnet synchronous servo system [J]. Journal of Nanjing aerospace and aeronautics University,.2004, 36 (2).

[5] Sun Sujuan. The anti aliasing active damping control strategy for broadband adaptable [J]. "Journal of China Electrical Engineering".2015, 35 (20): 5310-5316.

[6] Zhao Zilong. Voltage source converter dual harmonic suppression based on improved space vector pulse width modulation [J]. "power system automation". 2013, 37 (8): 112-116. 
[7] Chenna. Development and application of model controller of microgrid [J]. "protection and control of power system". 2015 (11): 115-120.

[8] Peng Jintao. Technology development and application of rotating diode non conduction monitoring system for large imported rotary brushless exciter,.2014 annual meeting of China Electric Engineering Society, 2014. 